Research Article

\title{
Evaluation of Agricultural Supply Chain Effects and Big Data Analysis Based on Internet of Things Technology
}

\author{
Qingjun Fang $\mathbb{D I}^{1}$ and Cai Su${ }^{2}$ \\ ${ }^{1}$ School of Management Engineering, Qingdao University of Technology, Qingdao, Shandong 266061, China \\ ${ }^{2}$ Logistics Management Teaching and Research Office, Qingdao Huanghai University, Qingdao, Shandong 266555, China \\ Correspondence should be addressed to Qingjun Fang; fangqingjun@qut.edu.cn
}

Received 9 September 2021; Revised 21 October 2021; Accepted 23 October 2021; Published 16 November 2021

Academic Editor: Gengxin Sun

Copyright (c) 2021 Qingjun Fang and Cai Su. This is an open access article distributed under the Creative Commons Attribution License, which permits unrestricted use, distribution, and reproduction in any medium, provided the original work is properly cited.

\begin{abstract}
Building an information-based rural economic supply chain is an effective way to implement the rural revitalization strategy. The construction of China's agricultural product logistics market is still very backward, mainly including imperfect information system and high cost of intermediate circulation links. How to optimize the supply chain of agricultural products, ensure the normal supply of agricultural products quality and quantity in the market, improve people's living standards, and achieve the purpose of improving benefits is an important topic to be studied today. Aiming at the problems of traditional agricultural products supply chain, the application of Internet of Things technology in supply chain can be effectively solved. Therefore, this paper proposes the research on the environmental effect evaluation of rural economic supply chain based on Internet of Things technology and proposes to build a wireless sensor network for agricultural products for the collection and exchange of agricultural products information. At the same time, RFID (radio-frequency identification) electronic tags integrated with sensors are applied in other links of the supply chain. The experimental results show that the construction of the rural economic supply chain system based on Internet of Things technology can effectively optimize the rural economic supply chain, realize efficient information sharing, improve the quality of agricultural products, reduce the cost of each link of the supply chain, improve the competitiveness of rural supply chain, and promote the good development of rural agricultural products and rural economic supply chain environment.
\end{abstract}

\section{Introduction}

The rural revitalization strategy is an important part of promoting the structural reform of the agricultural supply side and promoting the process of rural agricultural modernization. The effective way to realize this strategy is to integrate Internet of Things and information technology into the rural economic supply chain and build an agricultural intelligent supply chain on the basis of Internet of Things. There are differences between rural economy and rural agricultural products. The improvement and diversification of rural agricultural products are the basis of rural economic development. If agricultural products are only single development, it is difficult for rural economy to get rid of the existing state [1]. In the traditional rural agricultural product supply chain, there are many links and processes between the producers of agricultural products and the downstream of the supply chain. The information exchange between the two is hindered, and the matching degree is very low so that the producers of agricultural products lack awareness of the corresponding market demand and produce fewer types of agricultural products, which cannot meet the market demand. The final transaction of agricultural products is often carried out at a price much lower than the average market price [2, 3]. Because rural farmers have limited sales channels of agricultural products, it is difficult to directly contact consumers of agricultural products through individual strength. Even though direct transactions can be conducted with consumers through traditional farmers' markets and other channels, their transaction costs are gradually superimposed. The production scale and sales mode of individual farmers have little impact on the whole 
traditional agricultural product supply chain, and it is impossible to fundamentally optimize the agricultural product supply chain [4]. Therefore, in order to achieve the sustainable development of rural economy, the government and agricultural enterprises need to optimize and integrate the industrial layout and supply chain of rural agricultural products, reduce the information transmission links in the supply chain, and avoid information distortion [5].

In view of the above problems, this paper puts forward the research on the environmental effect evaluation of rural economic supply chain based on Internet of Things technology. This paper believes that the information transmission in the supply chain is the key of the Internet of Things supply chain. Therefore, it is proposed to build a wireless sensor network for agricultural products production in the sensing layer of the Internet of Things and apply RFID electronic tags integrating sensors in other links of the supply chain. This paper is mainly divided into three parts. The first part expounds the development of agricultural products supply chain and the development of Internet of Things technology in the supply chain. The second part is the construction of rural economic supply chain environment based on Internet of Things technology and expounds the system structure of rural economic supply chain based on Internet of Things technology. The third part is to test the performance of wireless sensor networks for production of agricultural products based on the Internet of Things, and collect, analyze, and evaluate the data of rural economic supply chain environment based on the Internet of Things.

This paper puts forward the research on the environmental evaluation of rural economic supply chain based on Internet of Things technology and constructs the rural economic supply chain environment based on Internet of Things technology. Internet of Things technology integrates it into the rural economic supply chain. In the whole supply chain system, the collection, analysis, and exchange of information and data play an important role. In view of this, this paper proposes to build a wireless sensor network for agricultural products in the sensing layer of the Internet of Things system and apply RFID electronic tags and sensors to other links of the supply chain so as to realize the detection and collection of physical data related to agricultural products.

\section{Related Work}

Since the 1990s, the global agricultural products market has entered the stage of oversupply, people's demand for agricultural products is also changing, and people pay more and more attention to service quality, safety of agricultural products, and environmental protection $[6,7]$. This makes many rural enterprises and governments begin to add new types of agricultural products and forms of production and consumption according to the actual situation of rural areas, which promotes the emergence of agricultural product supply chain [8]. In foreign countries, the research on agricultural product supply chain management focuses on agricultural product quality assurance, information technology management, value chain analysis, and general cooperation $[9,10]$. In the early years, some scholars proposed to increase the food supply chain on the basic supply chain and pointed out that the management of food supply chain, that is, a series of organizations such as agricultural products and food production and sales. It needs to implement the vertical integration operation mode if they want to ensure the quality and safety of agricultural products and corresponding food while reducing their logistics costs. And, this model has been widely used in many countries with relatively developed agricultural production [11-13]. The research on agricultural product supply chain in China started relatively late and is still in the development stage. According to the analysis of the relationship between agricultural industrialization and agricultural product supply chain, some scholars put forward that the construction and management of China's agricultural product supply chain needs the government to provide corresponding necessary technical support while gathering farmers' participation [14]. Some scholars also believe that enterprises play a core role in the agricultural product supply chain [15]. Other scholars have provided countermeasures to improve the status of vulnerable small production organizations in the agricultural product supply chain [16]. This shows that the supply chain of agricultural products is an important direction for the development of domestic agricultural products industry. Although it continues to develop, there are still many problems to be solved.

The application of the Internet of Things in the supply chain is mainly based on RFID and sensors. In China, most of the research is on the benefits after the implementation of the Internet of Things, the impact of the application efficiency of all links of the supply chain, and the information traceability of agricultural products $[17,18]$. Some scholars have designed an agricultural product information management system based on RFID technology and public key so as to trace the information of agricultural products in the supply chain [19]. Other scholars have proposed the application of RFID technology in the field of animal traceability and health monitoring [20]. Some scholars proposed to introduce the RFID technology into the food supply chain to trace and share information from food production and processing to final sales and consumption, so as to realize the role of food safety supervision [21]. However, at present, the application of the Internet of Things in the supply chain is still developing. With the development of information technology and science and technology, it also provides more directions and possibilities for the integration of the Internet of Things and the supply chain, so as to achieve the goal of the application of the Internet of Things technology in all links of the supply chain from point to line and finally to area.

\section{Construction of Rural Economic Supply Chain Environment Based on Internet of Things Technology}

The introduction of Internet of Things technology into the rural agricultural product supply chain can effectively solve the problems existing in the traditional rural agricultural 
product supply chain and promote the optimization of agricultural product industrial layout and supply chain [22]. Figure 1 shows the block diagram of rural economic supply chain system based on Internet of Things technology. According to the structure of Internet of Things technology, the system can be divided into three parts: perception layer, network layer, and application layer.

The sensing layer mainly detects the growth and production of agricultural products in real time through various sensors such as temperature and humidity, so as to understand and control the state of agricultural products at any time. Then, the growth information of corresponding agricultural products is recorded and obtained through electronic label sensors, so as to simplify the production and processing of agricultural products [23-25]. At the same time, in the retail and consumption links of the supply chain, consumers can also obtain the relevant information of agricultural products in all links through the electronic labels of agricultural products. The network layer is to transmit the agricultural product-related information obtained in the perception layer, that is, the origin, processing mode, market price, and other information of agricultural products to the information sharing platform, so as to achieve the purpose of agricultural product information sharing. Through the application layer, the producers of agricultural products can adjust the planting plan and layout of agricultural products in the future according to the feedback information of market demand. The machining center can also adjust the machining progress according to the machining feedback information $[26,27]$. The distribution department can reasonably allocate vehicles according to the obtained road information. Consumers can easily and quickly understand the relevant information of agricultural products. Government regulators can implement corresponding market regulation measures according to the trend of market price changes.

From the perspective of the rural economic supply chain based on the Internet of Things, the supply chain includes the production of agricultural products, the processing of agricultural products, the circulation and storage of agricultural products, the circulation and transportation of agricultural products, and the retail consumption of agricultural products. As shown in Figures 2 and 3, it is the block diagram of each link of rural economic supply chain based on Internet of Things technology [28]. As can be seen from the figure, sensor technology plays an important role in the whole rural agricultural product supply chain based on Internet of Things technology and is the key to the collection, acquisition, transmission, and interaction of supply chain information. Therefore, this paper proposes to build a wireless sensor network for agricultural production in the sensing layer to effectively monitor the growth state of agricultural products. At the same time, RFID technology and sensors are integrated to improve the effectiveness and practicability of agricultural product information transmission.

3.1. Construction of Wireless Sensor Network for Agricultural Products Production Based on Internet of Things. To vigorously develop fine agriculture in rural areas, it is necessary to effectively supervise and regulate the growth environmental factors of agricultural products through science and technology, and wireless sensor networks can meet their needs [23]. As the basic unit in the network, the sensor includes four modules: data acquisition, processing, wireless communication, and energy supply, as shown in Figure 4. Sensor nodes can be deployed randomly within the monitored range of farmland, form a network in the form of self-organization, collect and preprocess the data, and send data feedback according to the instructions issued by the Farmland Monitoring Center. The position of sensor nodes can be determined by GPS positioning or by using the positioning algorithm of the node itself. Considering the field of rural farmland, sensor energy management, and communication security requirements, this paper selects wireless sensor mode in most cases, and adopts the cluster energy supply mode. The nodes in each cluster use solar panels to supply power, while the central node uses municipal power to supply energy. The communication mode is cluster cross double-chain communication mode combined with limited ad hoc network mode. When the cluster head node is abnormal and cannot maintain normal operation, it can transmit data to nearby cluster heads through the ad hoc network. As shown in formula (1), the network energy consumption EC formula is as follows:

$$
\begin{aligned}
E C(R)= & p_{0, \text { proc }}\left(t_{0, \text { seup }}+t_{0, a c q}+t_{0, \text { proc }}\right)+p_{0, \text { tran }} t_{0, \text { tran }} \\
& +\sum_{k=1}^{H 1}\left\{p_{k, \text { proc }}+t_{k, \text { proc }}+p_{k, \text { tran }} t_{\text {tran }}\right\}
\end{aligned}
$$

where $R$ represents a routing path containing $k$ sensor nodes, its establishment time is expressed as $t_{0 \text {, seup }}$, and the corresponding power consumption is expressed as $p_{0,}$ proc. The time for processing data is expressed as $t_{k, a c q}$, the time for sending information to other nodes is expressed as $t_{t r a n}$, and the corresponding transmission power consumption is expressed as $p_{k, \text { tran }}$. The time when it returns to the route is expressed as $\mathrm{t}_{0 \text {, tran }}$.

As shown in formula (2), it is the calculation formula of network packet loss rate:

$$
p-\operatorname{loss}\left(p\left(v_{s}, v_{d}\right)\right)=\frac{\operatorname{loss}(e)}{\operatorname{packet}\left(p\left(v_{s}, v_{d}\right)\right)}
$$

where the total amount of data in $p\left(v_{s}, v_{d}\right)$ path is expressed as packet $\left(p\left(v_{s}, v_{d}\right)\right)$, and the total amount of loss is expressed as $\operatorname{loss}(e)$.

Location information plays a very important role in the operation of wireless sensor networks and will affect the transmission of information in the whole network. The location of sensor nodes in rural agriculture is variable. Considering the constraints of wireless sensor network nodes' power consumption, their own cost, and hardware conditions, trilateral measurement method and DV-hop algorithm are used to locate the location of sensor nodes in this paper. However, considering the relatively low accuracy of trilateral measurement method and DV-hop algorithm, this paper improves the algorithm based on it. 


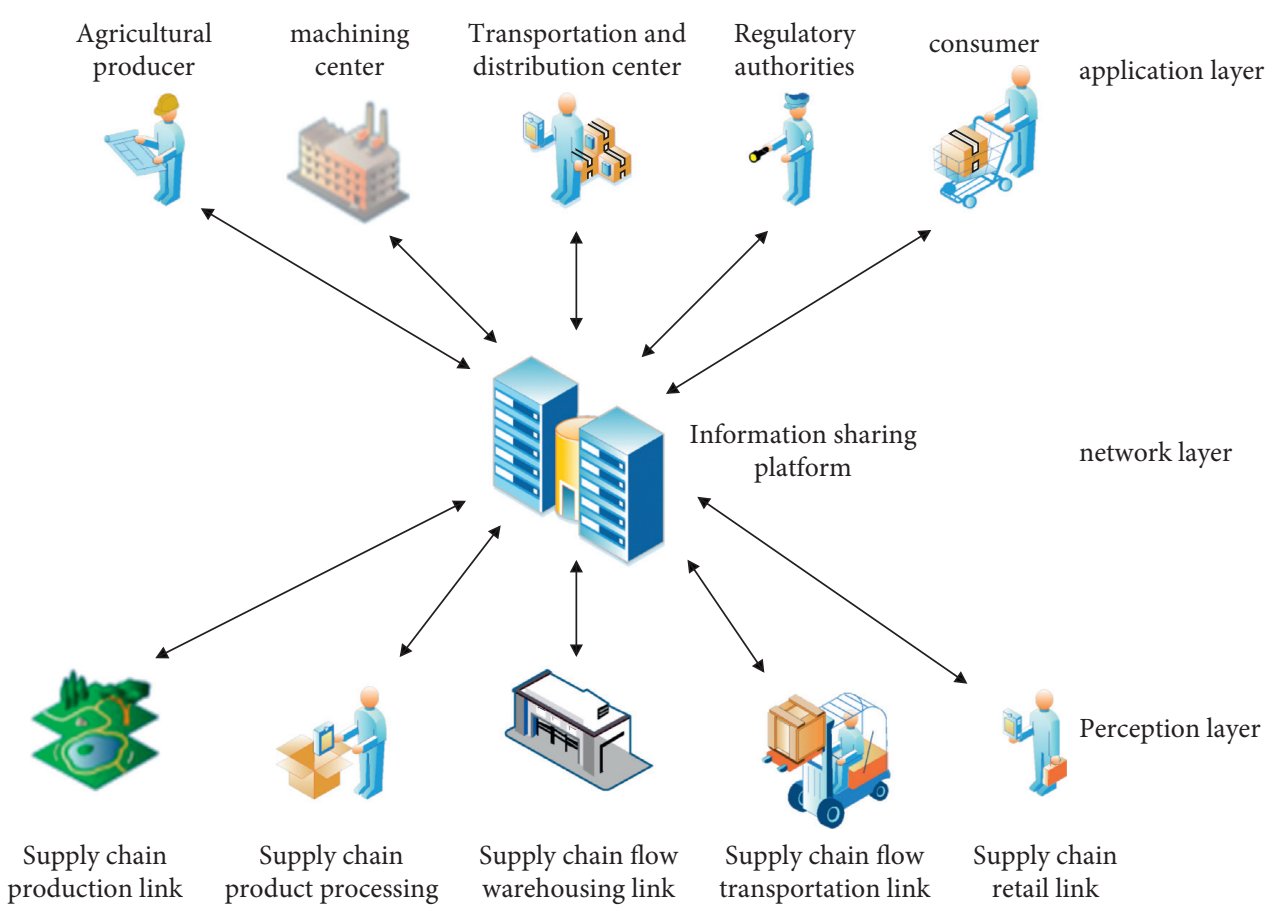

FIGURE 1: Block diagram of rural economic supply chain system based on Internet of Things technology.

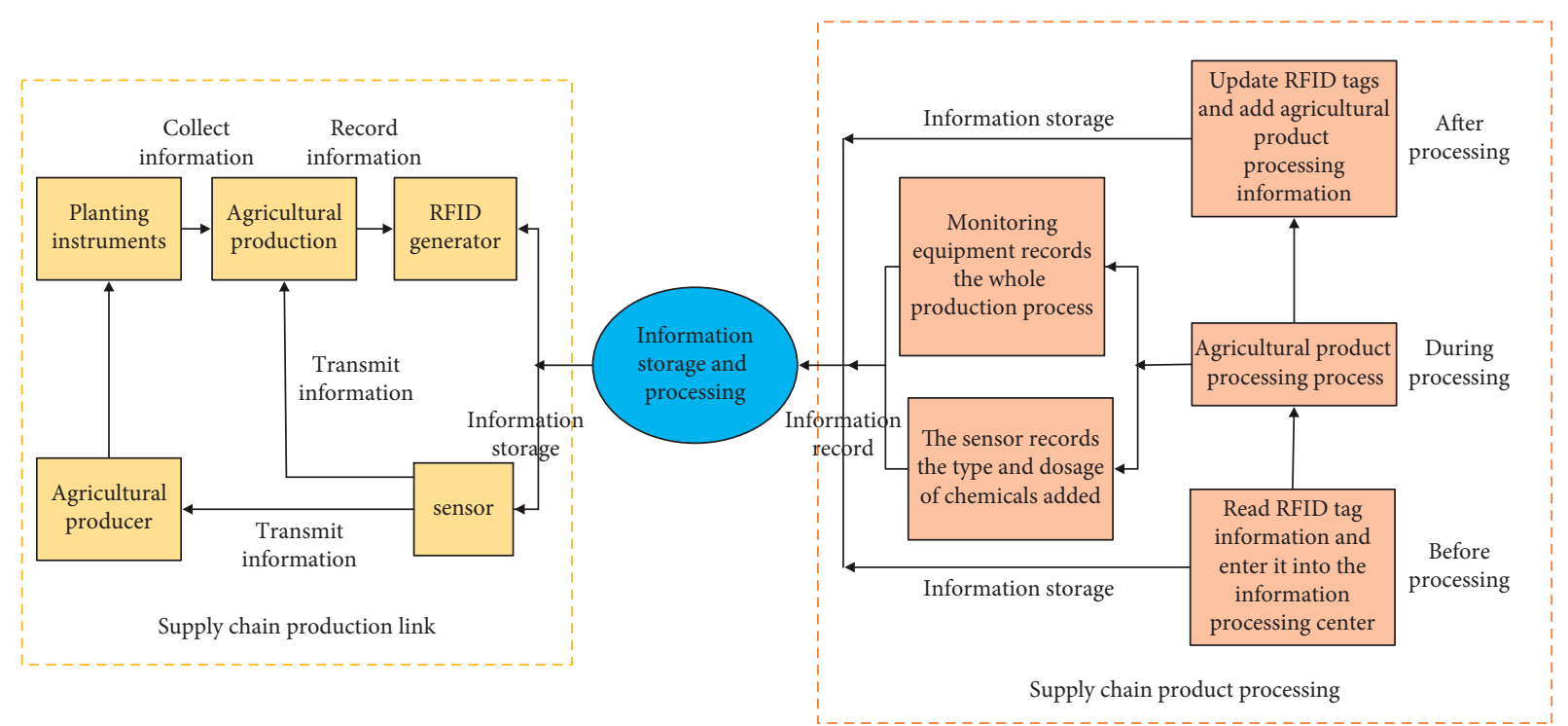

FIGURE 2: Production and processing of agricultural products in the rural economic supply chain based on Internet of Things technology.

Let the three known nodes be $\mathrm{A}, \mathrm{B}$, and $\mathrm{C}$ and their coordinates be $\left(\mathrm{x}_{\mathrm{a}}, \mathrm{y}_{\mathrm{a}}\right),\left(\mathrm{x}_{\mathrm{b}}, \mathrm{y}_{\mathrm{b}}\right)$, and $\left(\mathrm{x}_{\mathrm{c}}, \mathrm{y}_{\mathrm{c}}\right)$, and the distances between the unknown node $D$ and the three anchor nodes are expressed as $d_{a}, d_{b}$, and $d_{c}$, respectively. Take the three anchor nodes as the center of the circle and the distance value as the radius to draw the intersection of three circles, that is, the position of node $D$.

$$
\left\{\begin{array}{l}
\sqrt{\left(x-x_{a}\right)^{2}+\left(x-x_{a}\right)^{2}}=d_{a}, \\
\sqrt{\left(x-x_{b}\right)^{2}+\left(x-x_{b}\right)^{2}}=d_{b}, \\
\sqrt{\left(x-x_{c}\right)^{2}+\left(x-x_{c}\right)^{2}}=d_{b} .
\end{array}\right.
$$




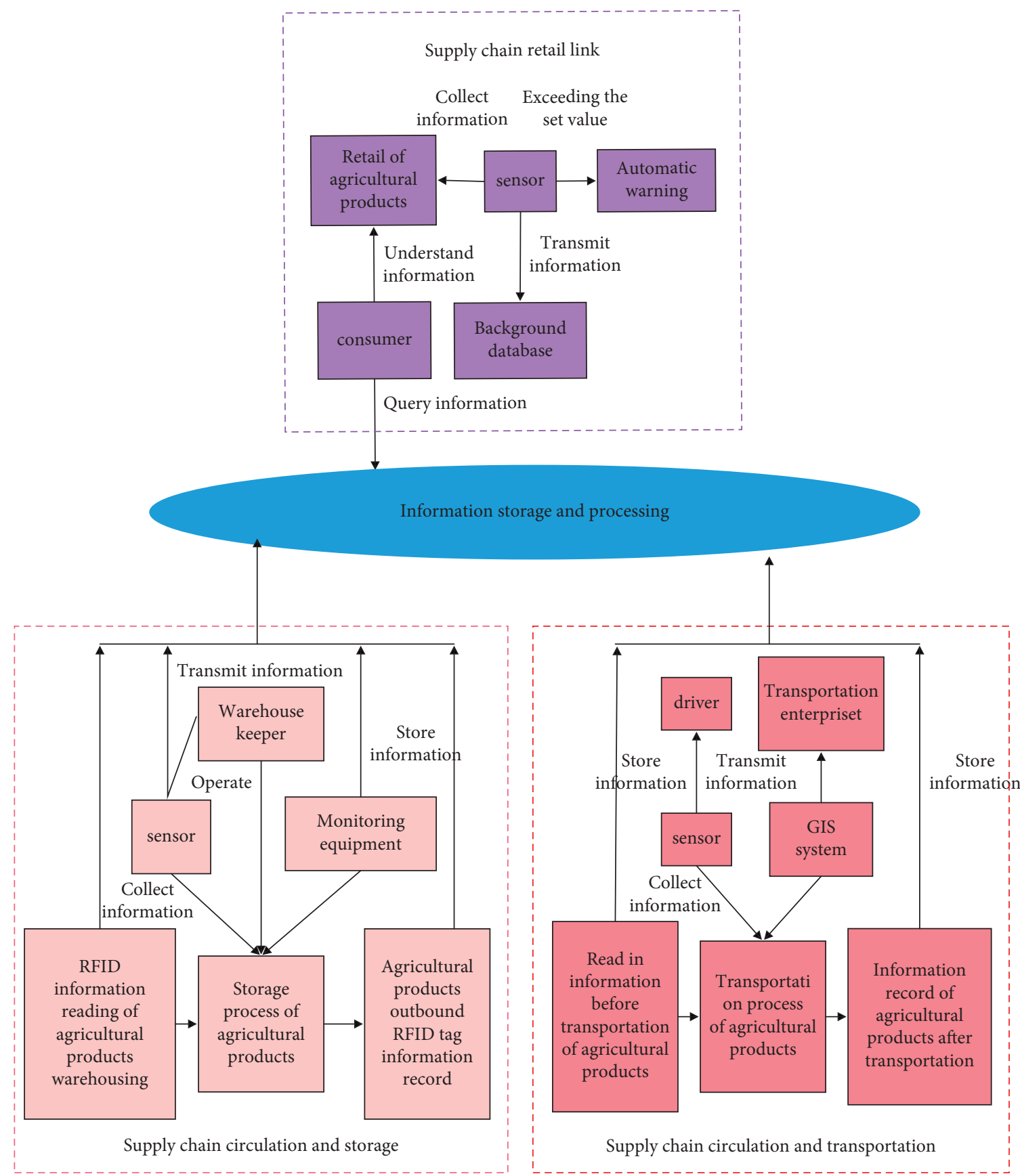

FIGURE 3: Circulation, storage, transportation, and retail of agricultural products in the rural economic supply chain based on Internet of Things technology.

According to the above formula, the coordinates of unknown node $D$ can be obtained, as follows:

$$
\begin{gathered}
{[x]\left[2\left(x_{a}-x_{c}\right) 2\left(y_{a}-y_{c}\right)\right]^{-1}\left[x_{a}^{2}-x_{c}^{2}+y_{a}^{2}-y_{c}^{2}+d_{c}^{2}-d_{a}^{2}\right][y]} \\
\quad=\left[2\left(x_{b}-x_{c}\right) 2\left(y_{b}-y_{c}\right)\right]\left[x_{a}^{2}-x_{c}^{2}+y_{b}^{2}-y_{c}^{2}+d_{c}^{2}-d_{b}^{2}\right] .
\end{gathered}
$$

According to the wireless sensor model, under the conditions of different transmission distance, antenna height, crop height, crop density, and different terrain, after analyzing the attenuation and packet loss rate of wireless RF signal, the calculation formula of information transmission connectivity of DV-hop algorithm is shown as follows:

$$
C M_{k, a_{i}}=\frac{n_{\text {receive }}\left(a_{i}, t\right)}{n_{\text {send }}\left(a_{i}, t\right)} 100 \%
$$

In time $t$, the number of signals received by node $k$ and sent by node $a_{i}$ is $n_{\text {receive }}\left(a_{i}, \mathrm{t}\right)$ expressed as $\left(a_{i}, t\right)$, and the number of signals sent by anchor node $a_{i}$ is expressed as $n_{\text {send }}\left(a_{i} t\right)$. 


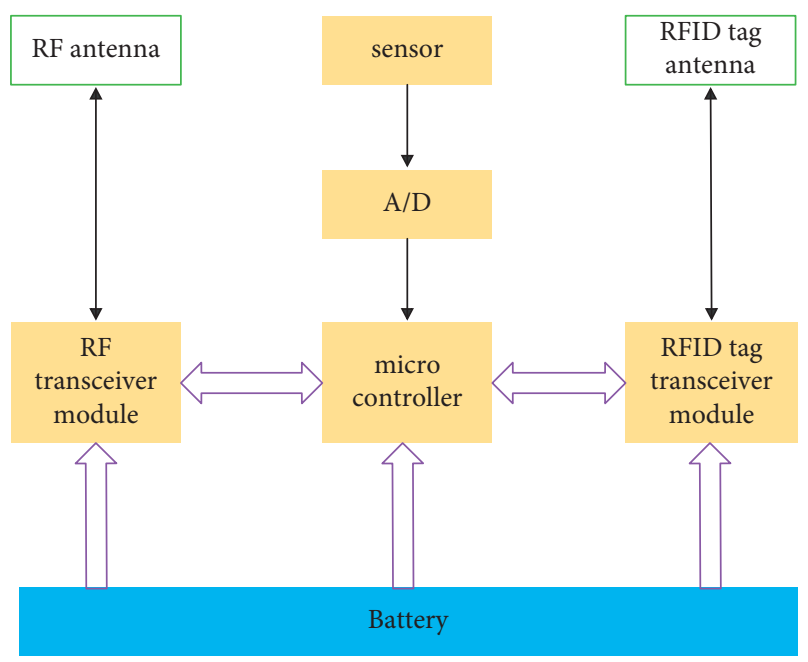

FIgURE 4: RFID electronic tag and wireless sensor fusion framework.

Each anchor node can estimate the average actual distance of each hop according to the corresponding location information of other anchor nodes and the number of hops apart, as follows:

$$
\text { HopSize }_{i}=\frac{\sum_{j \neq i}\left(x_{i}-x_{j}\right)^{2}+\left(y_{i}-y_{j}\right)^{2}}{\sum_{j \neq i} H_{j}},
$$

where the coordinate of signal node $i, j$ is expressed as $\left(x_{i}\right.$, $\left.y_{i}\right)$ and $\left(x_{j}, y_{j}\right)$, and the number of signal hops between them is expressed as $H j$. When the number of nodes is insufficient and the number of hops between location nodes and beacon nodes increases, there is a large error between the distance value of hops and the actual value, so it is necessary to improve the DV-hop algorithm.
According to formula (5), the average distance per hop of each beacon node can be obtained. Then, in this case, the deflooding routing method enables the unknown node to obtain the average distance per hop of the anchor node, and the distance value is weighted to obtain the final average distance per hop. Assuming that the distance from each beacon node to the unknown node contains the number of hops as $h_{1}, h_{2}, \ldots, h_{n}$, the weighted value of the average distance per hop of each anchor node is expressed as $W_{i}=\left(h /\left(h_{i}\right)_{n=1}^{n}\right)$, and the final average hop distance of the unknown node is expressed as $S$, and the calculation formula is shown as follows:

$$
S=\sum_{i=1}^{n} W_{i} \times \text { HopSize }{ }_{i}
$$

The distance is corrected as follows:

$$
\text { correction }_{i, j}=\frac{\left(H_{i, j}+H o p S i z e_{i} \times D i s_{i, j}\right) / H_{i, j}}{D i s_{i, j}},
$$

where $D i s_{i, j}$ represents the measured distance between anchor nodes, and $D i s_{i, j}=\sqrt{\left(x_{i}-x_{j}\right)^{2}-\left(y_{i}-y_{j}\right)^{2}}$. The final correction distance is shown as follows:

$$
\operatorname{disCorr}_{k, i}=\frac{\operatorname{dis}_{k, i}}{1+\text { Correction }_{k, i} \times h_{k, i}},
$$

where $\operatorname{dis}_{i}=\mathrm{S} \times \mathrm{h}_{i}$.

On the basis of trilateral ranging, an anchor node is introduced for positioning, and the position of unknown nodes is estimated through four anchor nodes. In order to improve its accuracy, a weighting factor is added to each group of positioning coordinates. The calculation formula is shown in formulas (10) and (11):

$$
\begin{gathered}
x_{E}=\frac{r_{B}}{r_{A}}+\frac{r_{C}}{r_{B}}+\frac{r_{D}}{r_{C}}+\frac{r_{A}}{r_{A}}+\frac{r_{B}}{r_{B}}+\frac{r_{C}}{r_{D}}+\frac{r_{A}}{r_{A}}+\frac{r_{B}}{r_{C}}++\frac{r_{D}}{r_{D}}+\frac{r_{A}}{r_{B}}+\frac{r_{C}}{r_{C}}+\frac{r_{D}}{r_{D}}, \\
y_{E}=\frac{\left(y_{1 E} / r_{B}+r_{C}+r_{D}\right)+\left(y_{2 E} / r_{A}+r_{B}+r_{C}\right)+\left(y_{3 E} / r_{A}+r_{B}+r_{D}\right)+\left(y_{4 E} / r_{A}+r_{C}+r_{D}\right)}{\left(1 / r_{A}+r_{B}+r_{C}\right)+\left(1 / r_{A}+r_{B}+r_{D}\right)+\left(1 / r_{A}+r_{C}+r_{D}\right)+\left(1 / r_{B}+r_{C}+r_{D}\right)} .
\end{gathered}
$$

3.2. RFID Electronic Tag and Sensor Fusion Framework. Traditional RFID technology can only meet the tracking needs of agricultural products, but cannot monitor the environment of agricultural products and the insurance status of agricultural products [24]. Therefore, this paper combines RFID and wireless sensor technology. As shown in Figure 4, it is the fusion framework of RFID electronic tag and wireless sensor. The fused sensor electronic tag not only has the function of general RFID electronic tag being read but also has the function of measuring specific physical parameters such as temperature and humidity.

In the rural economic supply chain system based on Internet of Things technology, the integrated RFID electronic label mainly reads the relevant data of agricultural products obtained by the sensor together with the RFID tag information through the setting of ZigBee network topology. After processing the read information data, upload it to the data processing center for further analysis and processing. In this process, we should pay attention to the energy consumption of wireless sensors integrating RFID electronic tags.

As shown in formula (12), K-bit data energy consumption:

$$
E_{T X}=E_{\text {elec }} \times K_{a m p}+\varepsilon \times K \times r^{2} .
$$

The energy consumption of $K$-bit data received by $\mathrm{RF}$ receiver is shown as follows: 


$$
E_{R X}=E_{\text {elec }} \times K,
$$

where, the electric energy required for $K$-bit data transmission is expressed as ETX, and the electric energy required for reception is expressed as ERX. The electric energy consumed in the process of encoding and modulating one bit data is expressed as $E_{\text {elec }}$. The coefficient of energy required to amplify the signal during unit data transmission in unit distance is expressed as $\varepsilon_{a m p}$. The distance between communication nodes is expressed as $r$.

According to the parameters in the above formula, it can be seen that the cost of receiving information is relatively high. The transmission distance of information should be shortened as much as possible, and the number of data operations in each message sending and receiving process should be simplified. In addition, wireless sensor protocols choose different routing methods, and their system consumption is also different. If the router node is selected for data forwarding, the energy consumption of the circuit at the data transmitting end of the routing node shall be amplified to minimize, as formula:

$$
E_{T X-a m p}(K, r)=\varepsilon_{a m p} \times K \times r^{2} .
$$

If a node with a distance of $n * d$ from the coordinator adopts the direct transmission protocol model, the energy consumed by $K$ bit data transmission is as follows:

$$
E_{1}=E_{T X}(K, r=n \times d)=E_{\text {elec }} \times K+\varepsilon_{\text {amp }} \times K \times(n \times d)^{2} .
$$

In the minimum energy multihop routing protocol transmission model, if there are $n-1$ nodes within the range of $n * d$ from the coordinator and the distance between nodes is $d$, the node far from the coordinator $n * d$ needs to send $n$ data with a distance of $d$ and receive $n-1$ data with a distance of $d$. According to formula (12),

$$
\begin{aligned}
E_{2} & =n \times E_{T X}(K, r=d)+(n-1) E_{R X}(K, r=d) \\
& =n \times\left(E_{\text {elec }} \times K+\varepsilon_{\text {amp }} \times d^{2}\right)+(n-1)\left(E_{\text {elec }} \times K\right) \\
& =K \times\left[(2 \times n-1) \times E_{\text {elec }}+\varepsilon_{\text {amp }} \times n \times d^{2}\right] .
\end{aligned}
$$

If and only if formula $(15)<(16)$, then

$$
\begin{gathered}
\varepsilon_{\text {amp }} \times n \times d^{2}<2 \times E_{\text {elec }}, \\
\frac{E_{\text {elec }}}{\varepsilon_{\text {amp }}}>\frac{n \times d^{2}}{2} .
\end{gathered}
$$

At this time, the direct transmission model consumes less energy than the multihop routing protocol model. On the contrary, the multihop routing protocol model consumes less energy than the direct transmission model. That is to say, when the data transmission distance is close or the wireless point energy is high, the direct transmission protocol saves more energy than the multihop routing protocol model.

\section{Result Analysis and Discussion}

4.1. Improved Algorithm Test of Wireless Sensor Network for Agricultural Products Production Based on Internet of Things.
The project adopts wireless sensor network technology to combine the Internet of Things with the collection of agricultural information. Intelligent measurement and control integrated application system for crop production based on Internet of Things. The purpose is to realize the automatic networking of wireless sensor network nodes in the target monitoring area, the real-time collection of environmental parameters affecting crop growth, and the data analysis and remote monitoring of host computer monitoring software. It is inevitable for agricultural development to replace traditional agriculture with intelligent precision agriculture based on the Internet of Things, which is more in line with China's national conditions.

In this paper, a rural orchard base is randomly selected for experimental test. Thirty nodes with a communication radius of $60 \mathrm{~cm}$ are selected and randomly distributed in the experimental range. According to the location information calculated by the sensor node, the number of tests is 120 , and the test data is the average value of 120 tests. Figure 5 shows the comparison results of the measured node positioning errors under different algorithms, different connectivity, and different anchor node ratios.

From the data in the figure, it can be seen that the improved DV-hop algorithm has better average error effect on the location of unknown nodes than the improved DV-hop algorithm, which shows that the improved DV-hop algorithm has better accuracy and can improve the performance of wireless sensor networks in rural economic supply chain.

4.2. Analysis on Environmental Assessment of Rural Economic Supply Chain Based on Internet of Things Technology. This paper uses the fuzzy comprehensive evaluation method to evaluate the different dimensions of rural economic supply chain environment based on Internet of Things technology. As shown in Figure 6, the competitiveness of each link of the rural economic supply chain and the overall supply chain before and after the use of the Internet of Things is compared.

From the data comparison in the figure, it can be seen that, after the Internet of Things is introduced into the rural economic supply chain, both the overall supply chain competitiveness of agricultural products and the competitiveness of all links of the supply chain are improved. The improvement of the competitiveness of agricultural production links is not obvious, mainly because in the early stage of building, a rural economic supply chain environment based on Internet of Things technology, the construction of wireless sensor networks, RFID electronic tags, and the maintenance of corresponding systems all need the process of cost digestion, which affects the improvement of the competitiveness of production links to a certain extent. The competitiveness of processing links, storage links, transportation links, and retail links has been significantly improved, which shows that the rural agricultural products supply chain based on Internet of Things technology can effectively and quickly share agricultural products, storage, transportation, and other relevant information so that all links of the supply chain can actively adjust according to the 


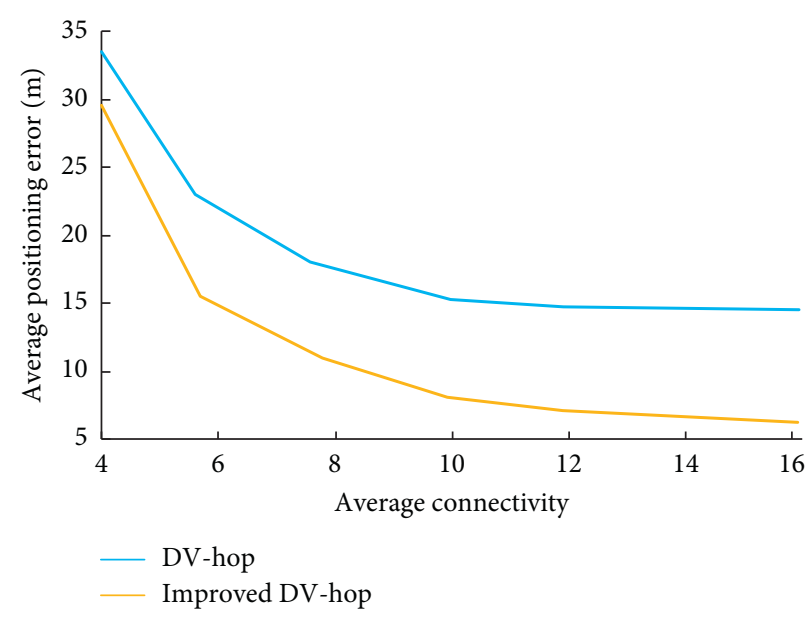

(a)

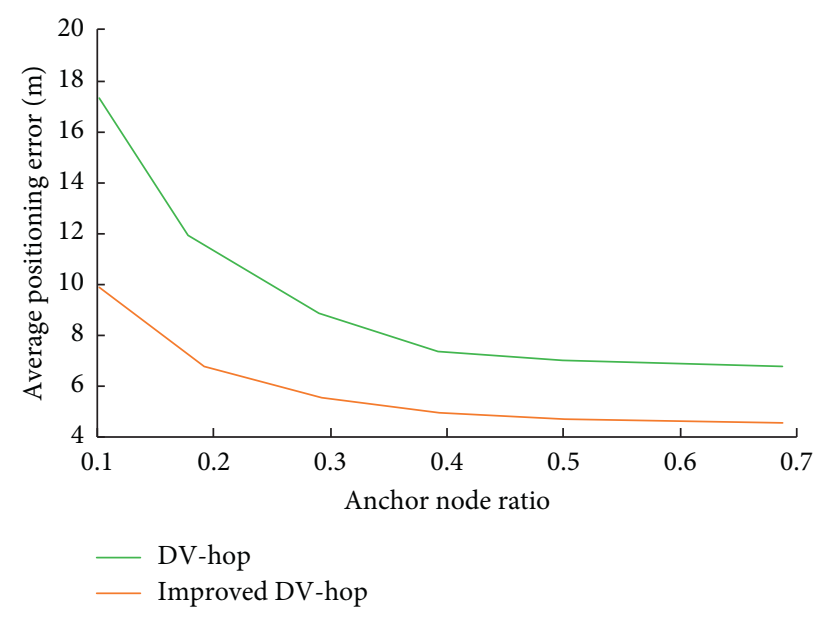

(b)

FIGURE 5: Comparison results of node positioning errors measured under different connectivity and different anchor node proportion.

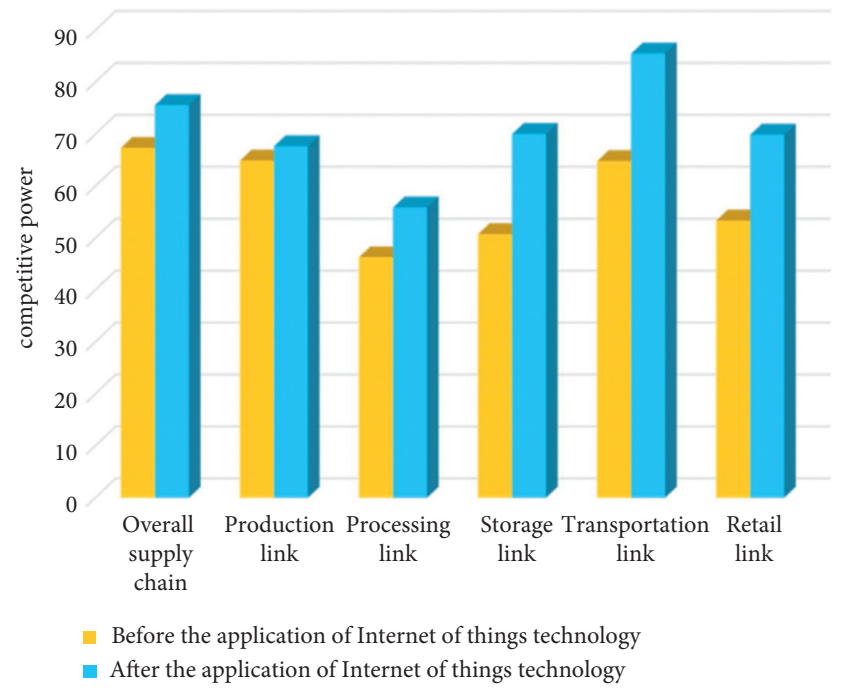

FIGURE 6: Comparison results of the competitiveness of each link of the rural economic supply chain and the overall supply chain before and after the use of the Internet of Things.

corresponding information and improve work efficiency. The supply chain of rural economy has been optimized. At the same time, it also shows that the wireless sensor network in the supply chain and the wireless sensor integrating RFID electronic tag can effectively collect the relevant physical parameters in the process of agricultural product growth, production, processing, and transportation and process and analyze them in the data center.

Figures 7-11 shows the comparison results of the competitiveness of various indicators in the five dimensions of the rural economic supply chain environment based on Internet of Things technology. The five dimensions include the financial status of rural economic supply chain, supply chain business process, green agricultural products, customer service, and learning and innovation of the whole rural economic supply chain. There are corresponding subindicators under each dimension.

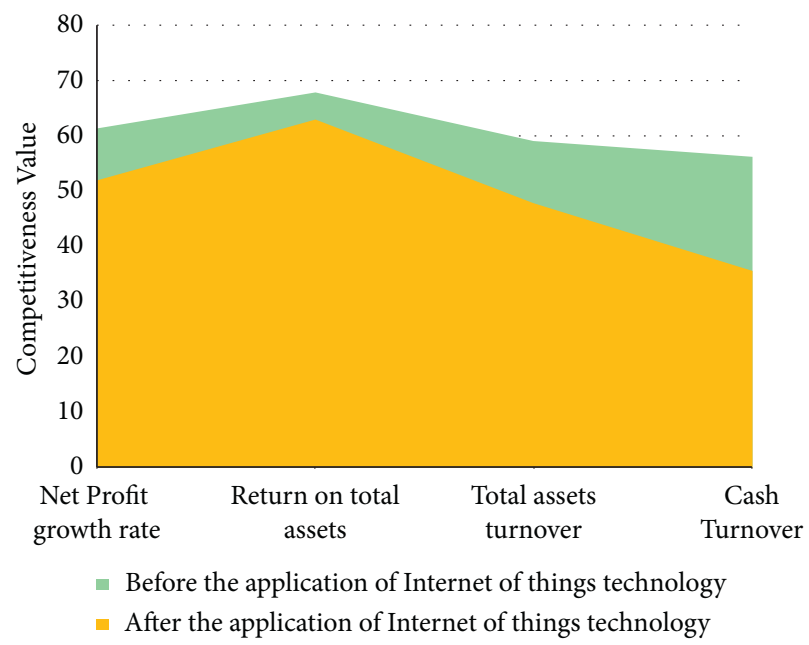

FIGURE 7: Supply chain competitiveness value of each subindicator of financial dimension.

It can be seen from the results in Figure 7 that, after the construction of rural economic supply chain based on Internet of Things technology, the competitiveness of each subindex in the financial dimension of the supply chain has decreased. This is mainly because the input cost in the early stage of system construction is still in the digestion stage, which has a certain impact on the net profit and cash flow of agricultural products.

As can be seen from Figure 8, the competitiveness of each subindex in the supply chain business process dimension has been improved. The application of Internet of Things technology and sensors has improved the information sharing degree of the rural economic supply chain, greatly reduced the operation cost of the supply chain, improved the work efficiency, and realized the optimization of the rural economic supply chain business process.

As can be seen from Figure 9, after the introduction of Internet of Things technology, the competitiveness of various subindicators of the green dimension of rural 


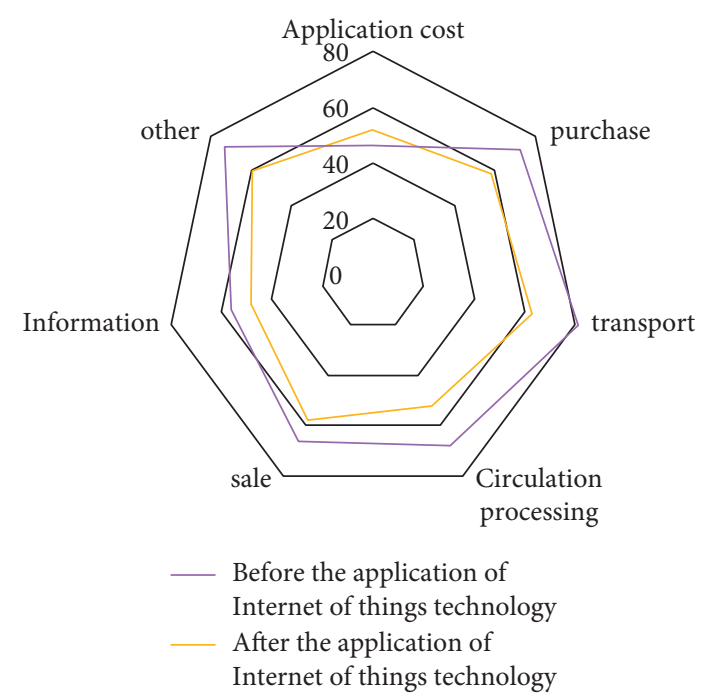

FIgURE 8: Competitiveness of subindicators in supply chain business process dimension.

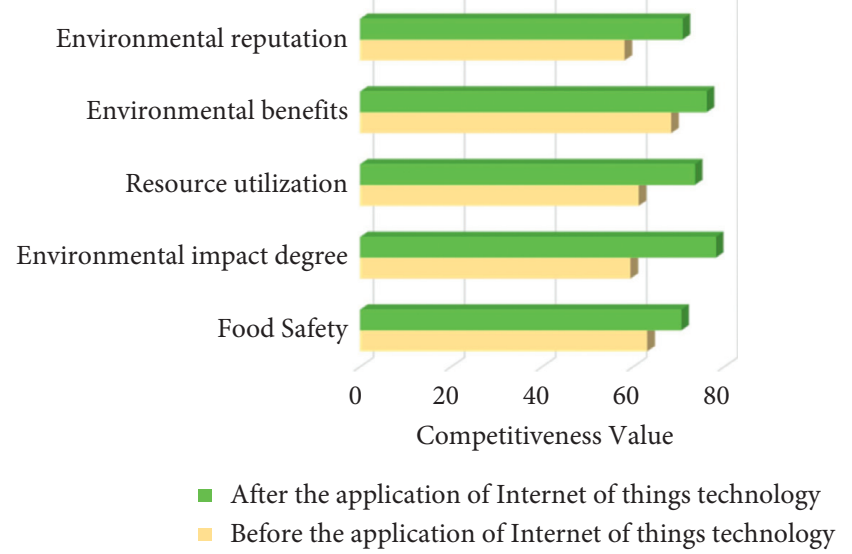

FIgURE 9: Comparison of competitiveness values of subindicators of green dimension.

agricultural products has also been improved. This is mainly because in the whole supply chain, Internet of Things technology and sensors can realize the whole process supervision of agricultural products and improve the quarantine degree of agricultural products. At the same time, the growth state of agricultural products is monitored by sensors, which effectively promotes the green degree of agricultural products and reduces the waste rate of agricultural products so as to reduce the pollution to the environment.

As can be seen from Figure 10, the application of Internet of Things technology has effectively improved the customer service ability of rural supply chain. At the same time, rural supply chain has strengthened the control of agricultural product quality, improved the quality of agricultural products, and more in line with the needs of the market and consumers. At the same time, customers can also understand the relevant information of agricultural products through the integrated RFID electronic tag, which improves customers' trust and satisfaction, thus increasing the probability of customers' repurchase.

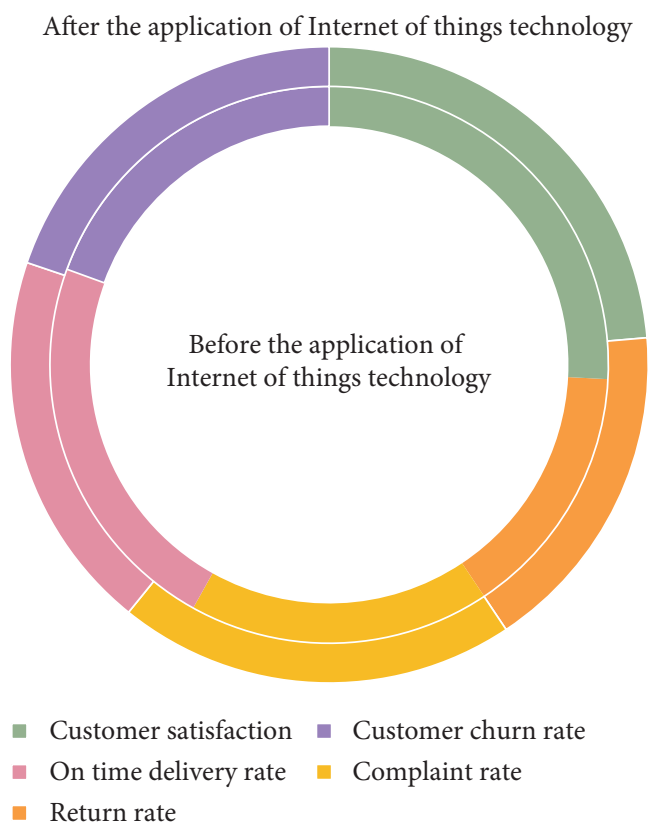

FIgURE 10: Competitiveness value of each subindex of the customer service index.

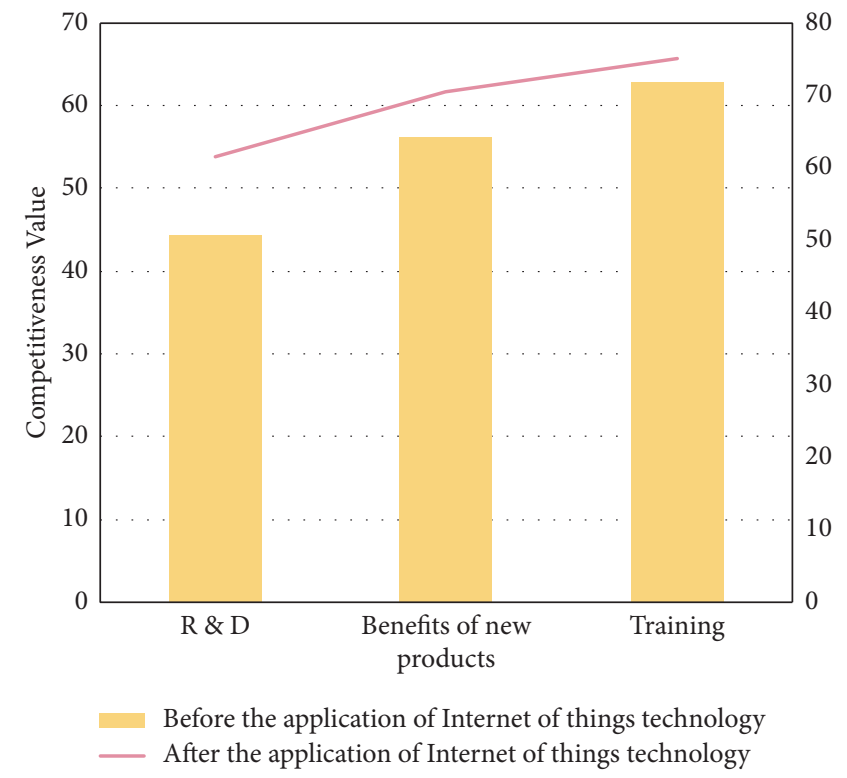

Figure 11: Comparison results before and after the competitiveness value of each subindex of learning and innovation index.

After constructing the rural economic supply chain environment based on Internet of Things technology, the innovation and learning ability of each link of rural supply chain has been significantly improved. On the surface, the informatization degree of the supply chain environment has been improved, which has driven the innovation ability of all links of the rural economic supply chain, shortened the emergence cycle of new agricultural products, increased the rural economy, made more rural workers get the opportunity to train skills, and increased more jobs. 
To sum up, the construction of rural economic supply chain system based on Internet of Things technology promotes the development of rural economy, improves the competitiveness of rural agricultural products, drives the development of rural agricultural products industry, optimizes the links of agricultural products supply chain, and makes the development of agricultural products and rural economy enter a state of benign development.

\section{Conclusion}

The traditional rural economic supply chain has been unable to meet the development of rural agricultural product economy due to many links, high cost, and noncirculation of information. With China's strong support for the rural revitalization strategy, how to optimize the rural economic supply chain has become a hotspot. This paper puts forward the research on the environmental evaluation of rural economic supply chain based on Internet of Things technology and constructs the rural economic supply chain environment based on.

The integration of IoT technology into the construction of rural economic supply chains enables the concrete use of IoT technology in economic activities. In the supply chain of the whole system, the collection, analysis, and exchange of information and data play an important role. In view of this, this paper proposes to build a wireless sensor network for agricultural products in the sensing layer of the Internet of Things system and applies RFID electronic tags with sensors in other links of the supply chain so as to realize the detection and collection of physical data related to agricultural products. The experimental results show that the improved DV-hop algorithm in agricultural wireless sensor networks is conducive to improve the accuracy of the network to confirm the location of unknown nodes, so as to improve the overall performance of the network. In addition, although the application of Internet of Things technology in the rural economic supply chain has limited improvement in the competitiveness of the overall supply chain in the early stage and even affects the financial income in the early stage, the competitiveness in other aspects has been significantly improved. This shows that the application of Internet of Things technology is conducive to optimize the rural economic supply chain process, improve the quality of agricultural products, reduce the cost of the supply chain, and realize the benign development of rural agricultural products industry and economy. However, the research algorithm in this paper still needs to be connected to the actual situation of rural agriculture. Therefore, in the future research, it is also necessary to integrate the technology into the rural economic supply chain for more verification, so as to promote the good development of rural agricultural products and rural economic supply chain environment.

\section{Data Availability}

The data used to support the findings of this study are available from the corresponding author upon request.

\section{Conflicts of Interest}

The authors declare that they have no conflicts of interest.

\section{References}

[1] S. Wolfert, L. Ge, C. Verdouw, and M.-J. Bogaardt, "Big data in smart farming - a review," Agricultural systems153, vol. 69, 2017.

[2] A. Tzounis, N. Katsoulas, T. Bartzanas, and C. Kittas, "Internet of Things in agriculture, recent advances and future challenges," Biosystems Engineering, vol. 164, pp. 31-48, 2017.

[3] A. I.-B. Ahmed and A. El-Sayed, "A new algorithm for cluster head selection in LEACH protocol for wireless sensor networks," International Journal of Communication Systems, vol. 31, no. 1, 2018.

[4] Y. Dong, B. Ding, and G. Zhang, "Quality safety traceability system based on agricultural product supply chain," Transactions of the Chinese Society of Agricultural Engineering, vol. 32, no. 1, pp. 280-285, 2016.

[5] M. A. Osama, "Dynamic relocation of mobile base station in wireless sensor networks using a cluster-based harmony search algorithm," Information Sciences, vol. 23, pp. 385-386, 2017.

[6] X. Chen, "Implementing the strategy of Rural Revitalization and promoting agricultural and rural modernization," Journal of China Agricultural University, vol. 35, no. 01, pp. 5-12, 2018.

[7] E. Reem, "Energy-efficient routing protocols for solving energy hole problem in wireless sensor networks," Computer Networks, vol. 114, 2016.

[8] R. Ranjan Swain and P. Bhoi, "Heterogeneous fault diagnosis for wireless sensor networks," Ad Hoc Networks, vol. 69, 2018.

[9] G. Aiello, I. Giovino, and M. Vallone, "A decision support system based on multisensor data fusion for sustainable greenhouse management," Journal of Cleaner Production, vol. 172, 2017.

[10] K. Pal and A.-U.-H. Yasar, "Internet of things and blockchain technology in apparel manufacturing supply chain data management," Procedia Computer Science, vol. 170, pp. 450457, 2020.

[11] A. H. Dehwah, S. Elmetennani, and C. Claudel, "UD-WCMA: An energy estimation and forecast scheme for solar powered wireless sensor networks," Journal ofNetwork and Computer Applications, vol. 90, 2017.

[12] A. Lh, X. B. Mei, and C. Bg, "Internet-of-things enabled supply chain planning and coordination with big data services: certain theoretic implications," Journal of Management Science and Engineering, vol. 5, no. 1, pp. 1-22, 2020.

[13] A. Syarif, A. Abouaissa, and P. Lorenz, "Operator calculus approach for route optimizing and enhancing wireless sensor network," Journal of Network and Computer Applications, vol. 97, 2017.

[14] K. Kondratjevs and V. Selmanovs-Pless, "Development of power supply management module for radio signal repeaters of automatic metering reading system in variable solar density conditions," Latvian Journal of Physics and Technical Sciences, vol. 53, no. 1, 2016.

[15] A. Motroni, A. Buffi, and P. Nepa, "Sensor-fusion and tracking method for indoor vehicles with low-density UHFRFID tags," IEEE Transactions on Instrumentation and Measurement, vol. 70, 2020. 
[16] A. Tyshchenko, "Building energy efficient wireless sensor networks," International Journal of Electronics and Telecommunications, vol. 63, no. 1, 2017.

[17] M. Noor-A-Rahim, M. O. Khyam, and X. Li, "Sensor fusion and state estimation of IoT enabled wind energy conversion system," International Journal of Electronics and Telecommunications, vol. 19, no. 7, 2019.

[18] M. O. Antonis, "Internet of things in agriculture, recent advances and future challenges," Biosystems Engineering, vol. 164, 2017.

[19] F. Miao, X. Lu, and B. Tao, "Design of rice traceability system based on WSN and RFID," Journal of Jishou University (Natural Sciences Edition), vol. 040, no. 003, pp. 33-38, 2019.

[20] F. Deng, P. Zuo, and K. Wen, "Low delay technology research of transmission line tower monitoring network integrating WSN and RFID," IEEE Access, vol. 99, p. 1, 2019.

[21] F. Tran, "Solar energy harvester for industrial wireless sensor nodes," Procedia Computer Science, vol. 105, 2017.

[22] X. Jiang, J. Xie, and W. Xing, "Design and research of wireless mobile diagnosis and treatment model based on RFID and WSN technology," Journal of Jiujiang University, vol. 35, no. 01, pp. 109-111, 2020.

[23] Y. . Jiang, "Combination of wearable sensors and internet of things and its application in sports rehabilitation," Computer Communications, vol. 150, 2019.

[24] C. Abreu, F. Miranda, and P. M. Mendes, "Smart contextaware QoS-based admission control for biomedical wireless sensor networks," Journal of Network and Computer Applications, vol. 88, 2017.

[25] P. Pong, K. Lam, and X. Liu, "Overview of spintronic sensors with internet of things for smart living," IEEE Transactions on Magnetics, vol. 99, 2019.

[26] S. Bin, G. Sun, N. Cao et al., "Collaborative filtering recommendation algorithm based on multi-relationship social network," Computers, Materials \& Continua, vol. 60, no. 2, pp. 659-674, 2019.

[27] G. Sun and S. Bin, "Router-level internet topology evolution model based on multi-subnet composited complex network model," Journal of Internet Technology, vol. 18, no. 6, pp. 1275-1283, 2017.

[28] G. Tian, S. Zhou, G. Sun, and C.-C. Chen, "A novel intelligent recommendation algorithm based on mass diffusion," Discrete Dynamics in Nature and Society, vol. 2020, Article ID 4568171, 9 pages, 2020. 